Proceedings of the 2002 IEEE/RSJ

Intl. Conference on Intelligent Robots and Systems

EPFL, Lausanne, Switzerland • October 2002

\title{
UKEMI: Falling Motion Control to Minimize Damage to Biped Humanoid Robot
}

\author{
Kiyoshi FUJIWARA Fumio KANEHIRO Shuji KAJITA Kenji KANEKO \\ Kazuhito YOKOI Hirohisa HIRUKAWA \\ Intelligent Systems Institute \\ National Institute of Advanced Industrial Science and Technology \\ 1-1-1 Umezono, Tsukuba 305-8568 JAPAN
}

\begin{abstract}
This paper investigates a method to minimize damage to a humanoid robot when it falls over to the ground. The strategy involves controlling the attitude of the robot while it is falling over so that it lands on the ground at one of shock-absorbing parts of the robot. A simulation study has confirmed that the proposed algorithm can make the robot land at specified shockabsorbing parts.
\end{abstract}

\section{Introduction}

In this paper, we examine how to minimize damage to a humanoid robot when it falls over to the ground. It is true that biped humanoid robots have several advantages including being able to step over obstacles and go up and down stairs. One of their disadvantages is that they may fall over and be damaged, and this is one of the fatal barriers to practical applications of humanoid robots. Humanoid robots cannot be accepted for use in society unless this problem is overcome.

Recently humanoid robotics is in an exciting stage[1, 2, 3] after the astonishing debut of the Honda P2[4]. Biped locomotion is being intensively studied, and the controller of Honda's robots is considered state of the art in the field. However, little has been reported on how to eval a humanoid robot to fall over safely and prevent the robot from being damaged. We have examined this problem, and we were motivated by the following observations.

Compared with a quadruped walking robot or wheeled one, the center of gravity of a biped-walking robot is located at a relatively high position and the size of the convex hull of the feet is smaller. A biped humanoid robot is essentially an unstable structure, and as such, little can be done to prevent the robot from falling over. In addition, the robot may be damaged seriously enough to prevent it from walking thereafter, since the impact between the robot and the ground may be large. The bigger the humanoid robot is, more serious the damage is. Consequently, it is important to address this problem.

The goal of our research is to prevent physical damage that would disable the locomotion ability of the robot, thus giving it a chance to stand up again[5]. Specifically, we have studied how to control the attitude of the humanoid robot while it is falling over to lessen the impact of its landing on the ground. The underlying idea here is similar to UKEMI in Judo, which means movements to moderate injury when a Judo player is thrown by a competitor.

This paper is organized as follows. Section 2 overviews our strategy for attitude control. In Section 3 , various falling motion controls are evaluated by simulations using the HRP-2 Prototype humanoid robot now under development. The integrated algorithm for the general falling motion control is described in Section 4. Section 5 concludes the paper.

\section{Strategy of UKEMI}

\section{$2.1 \quad$ Impact Points}

The structure of the human body has excellent properties to resist impact. Flexible skin and muscle can absorb impact as the first line of defense. They absorb most impulsive forces while a human is falling over before they are transmitted to the skeleton.

An example of artificial implementation of such a shock absorbing structure is the bumpers of automobiles. The bumper can absorb the impact of a collision by transforming and crashing itself. The same idea may be applied to a humanoid robot, and various passive structures can be used including spring-damper mechanisms, urethane foam and rubber. However, it would be ideal to cover the entire surface with such shock absorbers, but it is not practical since it would increase the weight of the 
robot too much and limit the movable ranges of the joints.

A reasonable solution to the problem is mounting absorbers at positions that are appropriate to receive the falling momentum and controlling the posture of the robot to hit the ground at those positions. This is the first strategy of our proposed algorithm. Using the analogy with a human, the first impact point could be its hip, knee, hand, elbow or back. Of these points, we gave first priority to the hip and knee, and decided to mount the most effective shock absorbers there. After the collision at the first impact point, the secondary points must be able to receive the transfer of the impact and break the force. Hands, elbows and the back were selected to be the secondary impact points.

\subsection{Introducing a squatting motion}

When a humanoid robot falls over, most of its potential energy is converted into the impact force. Since the potential energy is proportional to the height of its center of gravity, the impact force can be reduced if the center of gravity can be lowered before the robot completely falls over. To this end, the squatting down of the robot is incorporated into the falling motion, and then its potential energy can be dissipated by the damping torque generated at its joints. This is the second strategy.

\subsection{Decision to transfer to the falling mode}

Some feedback control is usually applied to control the biped locomotion of a humanoid robot. We have developed a feedback controller[6], in which the feet are used to generate recovering momentum and the position of the waist is controlled to generate an inertial force to maintain the posture of the body. When any of the control laws cannot work to recover the posture of the robot, the robot is going to fall over. Our proposed algorithm switches the mode of the controller from the walking mode to the falling mode when the controller encounters such a state. It is possible to enable the robot to estimate the position and attitude of its body using its gyro sensor and an accelerometer sensor. Based on current motion, the ground impact time is anticipated.

\subsection{Controlling the falling posture}

When the robot is falling forward, the squatting motion is incorporated and the robot is likely to hit the ground first within its knees then its hand. When the robot is falling backward, the same squatting motion is employed and it has a good chance of hitting the ground first with its hip then its back. The squatting motion is controlled to position the ground projection of the center of mass in the supporting polygon whose vertices consist of those of the feet and the first impact point as we expect. The first impact point is the knees when falling forward and the hip when falling backward. Then the momentum of the robot after the first impact can be decreased.

If the falling direction of the robot is not covered by the first impact point, when the robot is falling to a direction with no first impact point, we introduce a rotational motion along the yaw axis followed by the squatting motion. To generate the momentum along the yaw axis, the motions of the arms are used. When the robot has a waist joint, which allows yaw rotation of the upper body, its twisting motion is also effective to generate the momentum. The easier direction of the rotation is chosen to make the robot strike the ground at the first impact point. While the rotation along the yaw axis is generated, the squatting motion is used to lessen the ground reaction force. Then the friction between the feet and the ground is reduced, so the robot rotates more freely along the yaw axis. When the robot has reached the desired angle along the yaw axis, the squatting motion is used to increase the ground reaction force and the positions of the arms and waist are returned to their origins slowly. In this way, the robot can hit the ground at the first impact point with the desired posture of its arms and waist. Moving to the landing posture should be finished earlier than the landing prediction time. This is necessary to prepare to prediction error in the contact time.

\section{Case studies of falling motion controls}

\subsection{Simulation setup}

HRP-2 Prototype. For the target humanoid robot, we use the HRP-2 Prototype (HRP-2P for short), which was developed in the Humanoid Robotics Project (HRP for short), an R\&D project of the Ministry of Economy, Trade and Industry (METI) of Japan [1](See Figure 1). It has 30 degrees of freedom (Leg: $6 \times 2$, Arm: $6 \times 2$, Waist: 2, Head: 2, Hand: 2) (See Figure 2). The standing height of HRP-2P is about $150[\mathrm{~cm}]$, and its weight is about $60[\mathrm{~kg}][7]$.

From the structural features of HRP-2P, the impact points will be selected as follows. Impact absorbing structures for the first impact point using flexible material are mounted on the hip and knees whose links are considered tough. Damage to the robot is expected not to be serious when the robot lands on these points. Flexible materials are mounted on the surfaces of the elbows, wrists, and lower part of the back, and whose internal structures are considered tough. They were selected as the secondary impact points. Impacts at these points should be reduced 


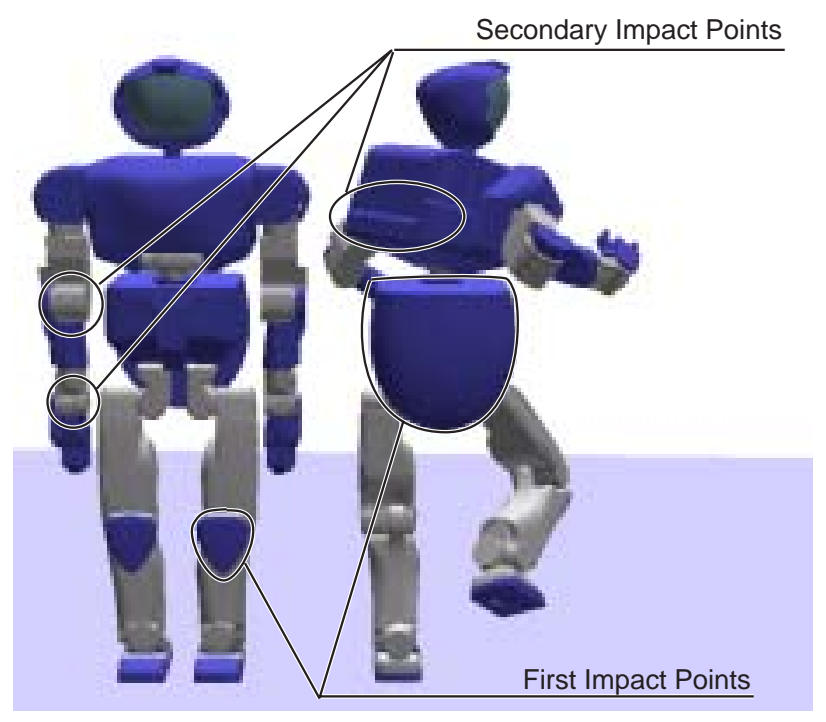

Figure 1: HRP-2 Prototype Humanoid Robot

to prevent the secondary impact points from being damaged. The structures of upper part of the back, head, front of the chest and tips of the hands are relatively fragile, and no impact force should be applied to them. Though the remaining parts may not be fragile structures, excessive impact should not be applied because no special protectors are attached there.

OpenHRP. We use OpenHRP[8] as the dynamics simulator. OpenHRP was developed in HRP for humanoid robots. It implemented as distributed objects using CORBA, whose servers include a dynamics simulator, collision checker and VRML-based model parser (See Figure 3). In a real robot, it is considered that the behavior of the tipping over process will difference in such factors as the measurement error of the field sensor, detailed shapes of the landing position, difference of the minute shape of the foot edge, delicate balance, etc. In addition, the momentum status when tipping over starts also varies.

\subsection{Falling Backward}

As the first study, we simulated HRP-2P falling over backward. Figure 4 shows a falling motion without any control, that is, the robot falls over with its arms at its sides. In the figure, snapshots of the falling humanoid are shown taken at every 0.5 second. Vertical straight-line segments from the floor in the figure show the contact forces from the floor. Generally, the extent of destructive impact is determined by the size of the applied force and the area. In this paper, the damage has been evaluated only by the speed and

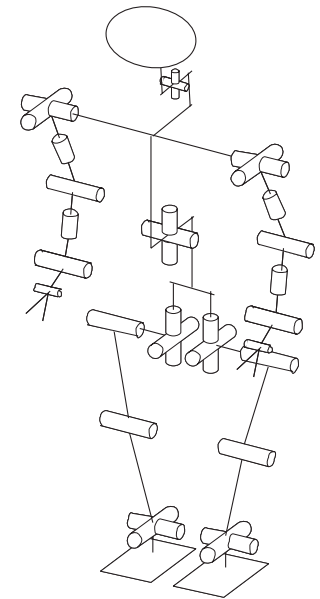

Figure 2: Joint Structure of HRP-2P

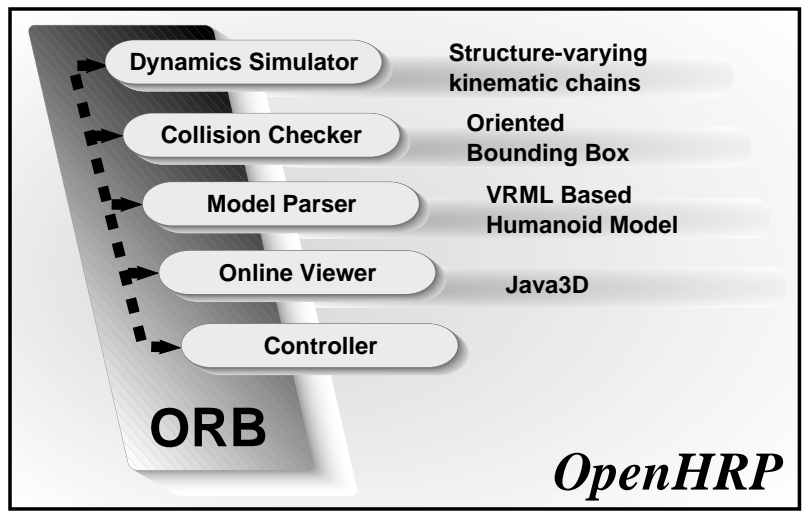

Figure 3: OpenHRP: Dynamics Simulator for humanoid robots

floor reaction in the moment of the landing.

A simulation of controlled falling is illustrated in Figure 5 . The snapshots are also taken at every $0.5 \mathrm{sec}-$ ond. The legs bend rapidly at the knees when the falling is started, the center of the mass of the robot is lowered, and its hip is stuck out. The supporting polygon made of the elbows, wrists and the lower part of the back is extended by spreading the arms to prevent the center of pressure moving to the upper part of the back. The center of the pressure is stopped at the hip, so the motion of the arms has not been made effective. Figure 6 is a comparison of the contact forces when falling over without control and with control. In the case without control, we can observe the impact to the back at $1.25[\mathrm{sec}]$. On the other hand, the back did not collide with the ground when our proposed control was used. It is acceptable though the impact is larger with the control since the 


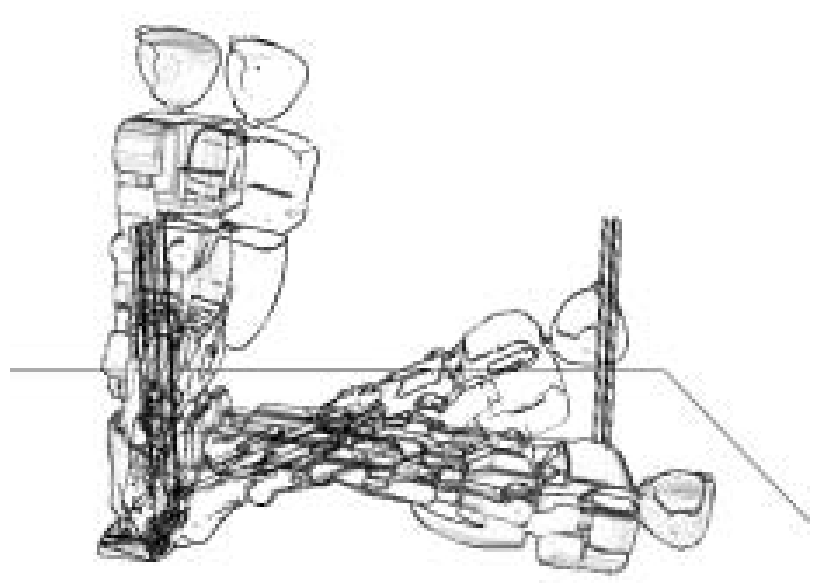

Figure 4: Simulation: Falling Backward (without control)

hip is designed to withstand hard impact.

\subsection{Falling Forward}

We also simulated the robot falling over forward. The result of the falling motion without any control is illustrated in Figure 7. These snapshots are also taken at every 0.5 second. We confirmed that the humanoid would land on the front of its chest.

The result of the simulation with control when falling over is illustrated in Figure 8. These snapshots are also taken at every 0.5 second. The robot rapidly lands on its knees after the falling started, and it lands on its forearms thereafter. Figure 9 shows that the knees received large impact. However, this is acceptable, since the knees are well protected by shock absorbing materials as they are first impact points. Also, the impact to the forearms is significantly less than that to the chest when striking the ground without control.

\subsection{Falling over to the right side}

The result of the simulation of falling to the right side without control is illustrated in Figure 10. The snapshots are also taken at every 0.5 second.

When falling over the robot keeps its arms down to the sides, it lands on the right arm, the center of pressure moves to the right shoulder, and the humanoid rolls back on to the upper part of its back.

The result of the controlled falling is illustrated in Figure 11. These snapshots are taken at zero, 0.5 and 0.7 second. Since the arms and the shoulders are not protected as are the other impact points, the robot needs to change quickly its body direction to land on its hip.

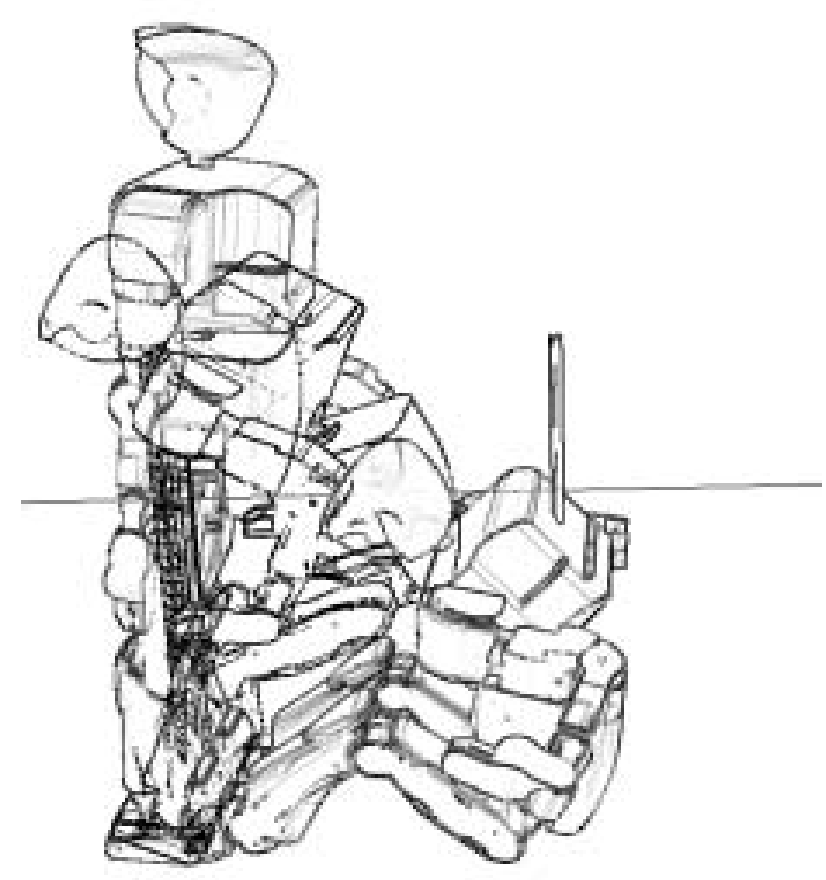

Figure 5: Simulation: Falling Backward (with control)

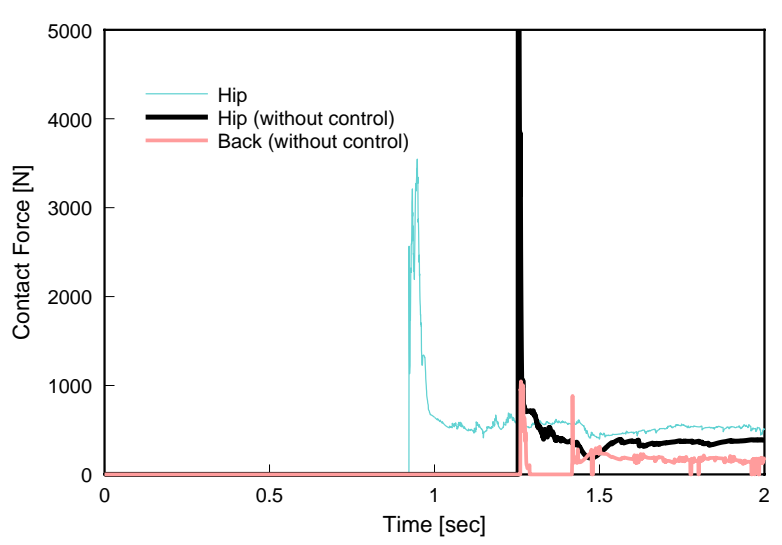

Figure 6: Contact forces when falling backward 


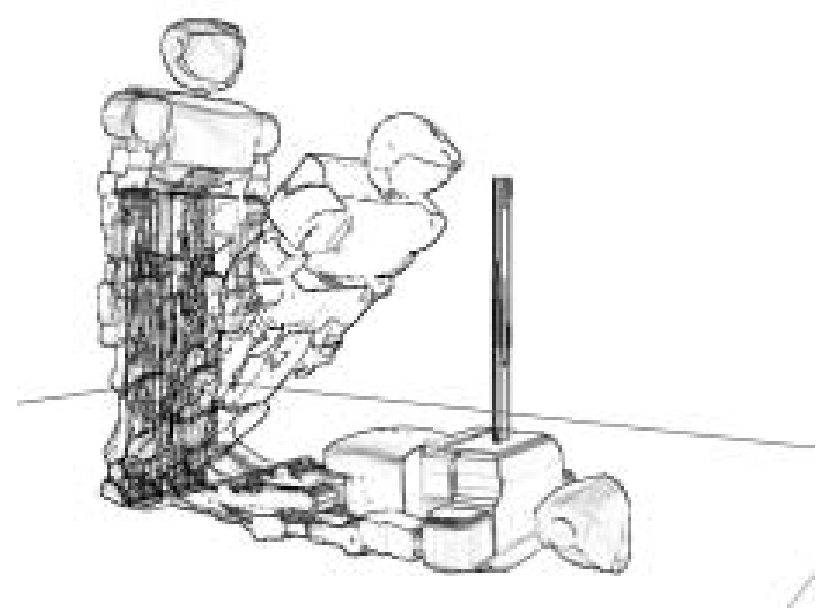

Figure 7: Simulation: Falling Forward (without control)

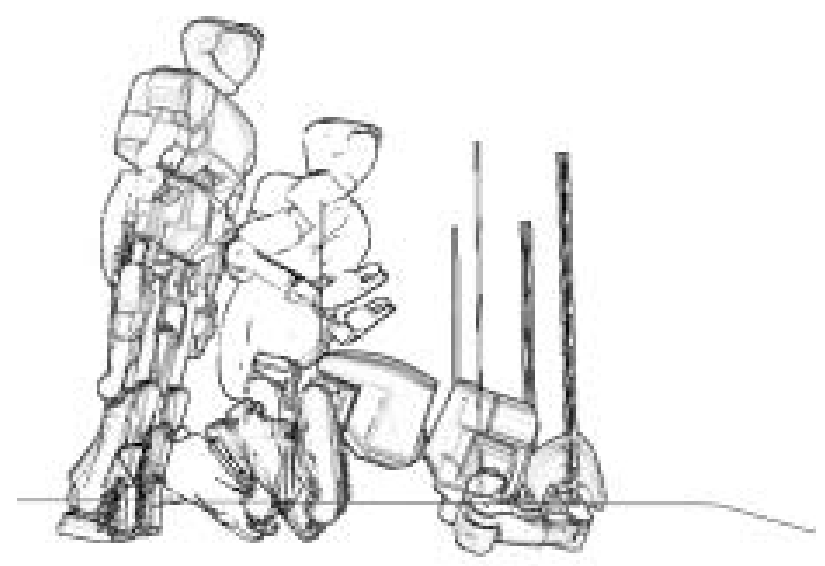

Figure 8: Simulation: Falling Forward (with control)

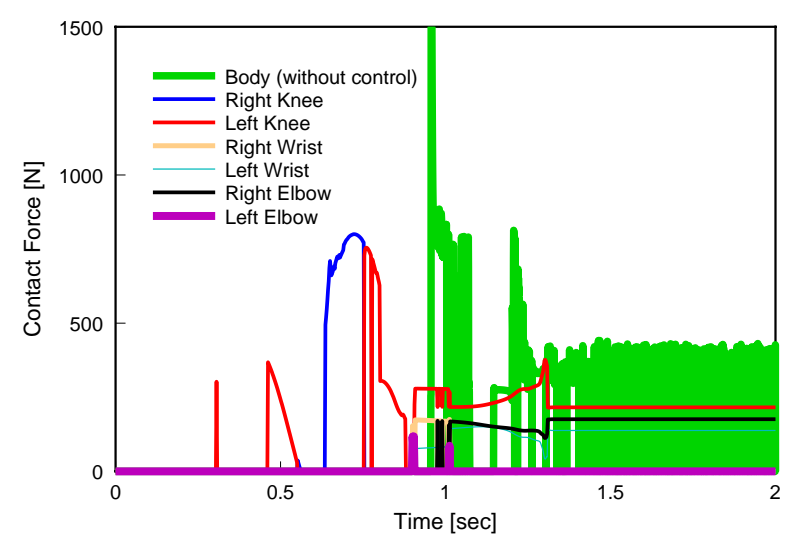

Figure 9: Contact forces when falling forward

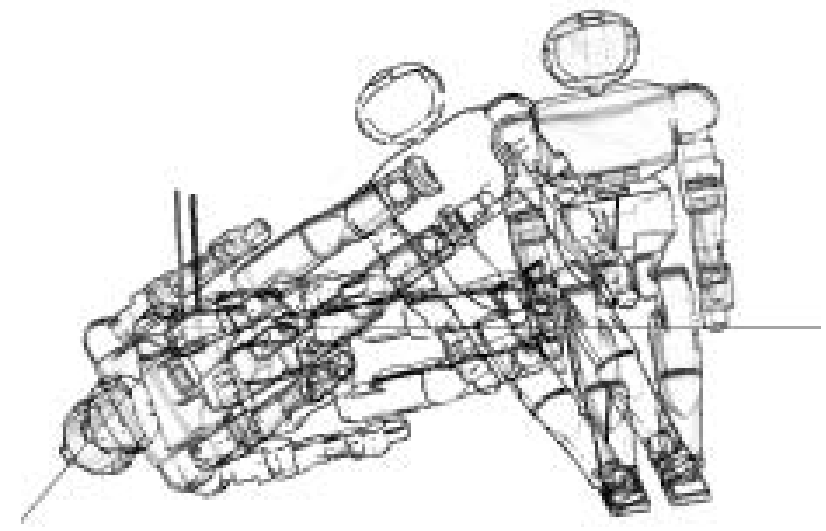

Figure 10: Simulation: Falling to the ight side (without control)

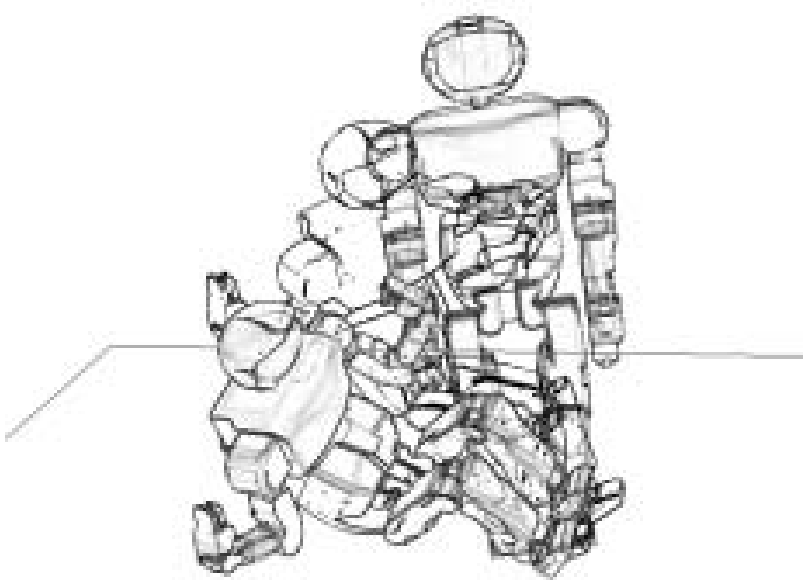

Figure 11: Simulation: Falling to the right side (with control)

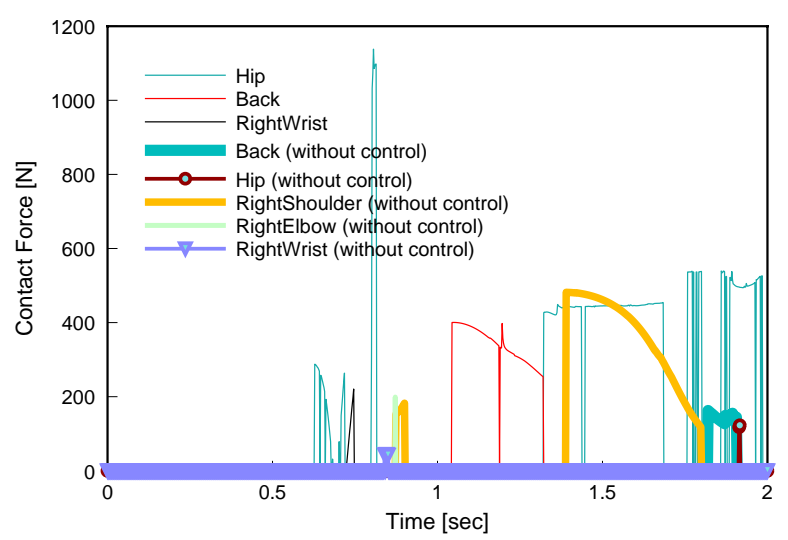

Figure 12: Contact forces when falling to the right side 


\section{The algorithm}

To control a humanoid robot when falling in various directions, we integrate the control methods discussed in the last section. The following UKEMI algorithm, which we had named after the similar protective Judo skill, can protect a humanoid robot from a possible damage of falling over, just like a skill of UKEMI.

\section{Algorithm: UKEMI}

1. As determined by the balance control system, transit into the falling mode when the attitude error of the humanoid exceeds a limit.

2. Identify the falling direction $D$ from the angular momentum of the robot along the roll and pitch axis and from the posture of the body.

3. Find the first impact point $H$ from $D$.

4. If $D$ is not within the accepted range of $H$, find the necessary rotation $R_{\text {yaw }}$ along the yaw axis, the required time $T_{\text {yaw }}$ for the rotation, and the expected time $T_{\text {land }}$ before landing.

5. If $T_{\text {yaw }}<\frac{T_{\text {land }}}{2}$, execute the rotation $R_{\text {yaw }}$ by moving the arms and the waist joints while making the robot squat to reduce the friction.

6. Predict the landing position from the supporting point on the feet, and modify the motions of the arms and the waist to make the relative velocity to the ground smaller while making the robot squat to increase the friction.

7. Make the robot squat to position the center of its gravity projected to the ground in a supporting polygon whose vertices consist of those of the feet and the first impact point.

8. When acceleration beyond a threshold is sensed, the robot has landed on the ground at the first impact points. Then move the arms and the waist to make the robot land at the secondary impact points.

9. When no movement is detected, stop the algorithm.

\section{Conclusions}

In this paper, we investigated how to minimize damage to a humanoid robot when it falls over to the ground. Several simulation results show that it is possible to land at specified parts when a humanoid robot falls down from on upright position. We have shown that effective impact absorption is possible, even if the shock-absorbing structure does not cover the entire body. In a future study, we plan more detailed examinations of the simulation results and falling experiment using real humanoid robots.

\section{References}

[1] Inoue, H., Tachi, S., Nakamura, Y., Hirai, K., et.al, "Overview of Humanoid Robotics Project of METI," Proc. Int. Symp. Robotics, pp.1478-1482, 2001.

[2] Nishiwaki, K., Sugihara, T., Kagami, S., Kanehiro, F., Inaba, M., and Inoue, H., "Design and Development of Research Platform for Perception-Action Integration in Humanoid Robot: H6," Proc. Int. Conference on Intelligent Robots and Systems, pp.15591564, 2000.

[3] Yamaguchi, J., Soga, E., Inoue, S. and Takanishi, A., "Development of a Bipedal Humanoid Robot - Control Method of Whole Body Cooperative Dynamic Biped Walking -," Proc. of the 1999 ICRA, pp.368374, 1999

[4] Hirai, K., Hirose, M., Haikawa, Y. and Takenaka, T., "The Development of Honda Humanoid Robot," Proc. of the 1998 ICRA, pp.1321-1326, 1998.

[5] Inaba, M., Kanehiro, F., Kagami, S., and Inoue, H., "Two-Armed Bipedal Robot that can Walk, Roll-over and Stand up," Proc. of the IEEE/RSJ Int. Conference on Intelligent Robots and Systems, pp.297-302, 1995.

[6] Yokoi, K., Kanehiro, F., Kaneko, K., Fujiwara, K., Kajita, S., and Hirukawa, H., "A Honda Humanoid Robot Controlled by AIST Software," Proc. Second IEEE-RAS Int. Conf. on Humanoid Robots, 2001.

[7] Kaneko, K., Kanehiro, F., Kajita, S., Yokoyama, K., Akachi, K., Kawasaki, T., Ota, S., and Isozumi, T., "Design of Prototype Humanoid Robotics Platform for HRP," (Paper submitted for IROS2002)

[8] Hirukawa, H., Kanehiro, F., Kajita, S., "OpenHRP: Open Architecture Humanoid Robotics Platform," Intr. Symposium on Robotics Research (ISRR), Melbourne, November 2001 (printing). 